\title{
FATORES PROGNÓSTICOS NO TRATAMENTO DOS TUMORES INTRAMEDULARES
}

\author{
Andrei Koerbel ${ }^{1}$, Claudio Esteves Tatsui ${ }^{1}$, Daniel Monte-Serrat Prevedello', \\ Ricardo Alexandre Hanel'2, César Vinícius Grande², Léo Fernando da Silva Ditzel', \\ João Cândido Araújo ${ }^{4}$, Affonso Antoniuk ${ }^{5}$
}

\begin{abstract}
RESUMO - Entre 1993 e 1999, foram analisados 35 pacientes submetidos a microcirurgia para remoção de tumor intramedular nos Serviços de Neurocirurgia do Hospital Nossa Senhora das Graças e do Hospital de Clínicas de Curitiba - PR. A população foi composta por $68,6 \%$ de pacientes do sexo masculino; a média de idade era de 32,9 anos. A localização tumoral mais frequente foi torácica em $40 \%$ dos casos, seguida pela região cervical e cervicotorácica em 25,7\%. Ao exame neurológico realizado entre 6 a 12 meses do pósoperatório, $42,9 \%$ dos pacientes demonstraram melhora, 34,3\% permaneceram estáveis e $22,9 \%$ dos casos apresentaram piora. A ressecção foi total em $57,2 \%$ dos casos e subtotal em $37,1 \%$. Em 5,7\% dos pacientes, foi realizada apenas biópsia para diagnóstico. 0 grau de ressecção completa foi maior nos ependimomas (13 de 17 casos) e menor nos astrocitomas (5 de 12 casos). Entretanto, o grau de ressecção e o tipo histológico não interferiram na morbidade pós-operatória. Fatores como sexo, idade e tamanho do tumor também não demonstraram importância na evolução após a cirurgia. A localização torácica do tumor foi associada a maior morbidade cirúrgica $(p=0,021)$.
\end{abstract}

PALAVRAS-CHAVE: tumor intramedular, microcirurgia, medula espinhal, ependimoma, astrocitoma.

Prognostic factors in the treatment of intramedullary spinal cord tumors

\begin{abstract}
Between 1993 and 1999, in the Divisions of Neurosurgery of the Hospital Nossa Senhora das Graças and the Hospital de Clínicas in Curitiba, Paraná, Brazil, 35 patients harboring intramedullary spinal cord tumors who were submitted to microsurgery were analyzed. There were 24 males $(68.6 \%)$ and average age was 32.9 years. The main location, with $40 \%$ of cases, was the thoracic level, followed by cervical and cervico-thoracic levels with 25.7\%. Neurological exam, carried out between 6 and 12 months after surgery, showed that $42.9 \%$ of patients improved, $34.3 \%$ were stable and $22.9 \%$ presented neurological worsening. Total resection was obtained in $57.2 \%$ of cases and subtotal in $37.1 \%$. In $5.7 \%$ of patients a biopsy was the accomplished procedure. Total resection was more often obtained among patients with ependymomas (13 out of 17) than with astrocytomas (5 out of 12). However, degree of resection and tumor histology did not interfer in postoperative morbidity. Factors as sex, age and tumor's size also did not demonstrate significance in predicting prognostic after surgery, whereas tumor in a thoracic level was associated with higher morbidity $(p=0.021)$.
\end{abstract}

KEY WORDS: intramedullary tumors, microsurgery, spinal cord, ependymoma, astrocytoma.

Os tumores intramedulares correspondem de 2 a $4 \%$ dos tumores do sistema nervoso central ${ }^{1}$ e a $10 \%$ dos tumores espinhais ${ }^{2}$, sendo os ependimomas e os astrocitomas os tipos histológicos mais frequentes ${ }^{1,3-6}$. Com o advento da ressonância magnética (RM) e com a evolução das modernas técnicas microcirúrgicas, os resultados pós-operatórios melhoraram significativa- mente. Embora os resultados cirúrgicos estejam diretamente relacionados ao estado neurológico pré-operatório do paciente e ao grau de malignidade do tumor. Quanto a outros fatores prognósticos, os dados são discordantes na literatura ${ }^{7-11}$. Neste estudo, são analisados fatores que eventualmente interfiram na ressecção e na morbidade cirúrgica.

\footnotetext{
Departamento de Neurocirurgia do Hospital Nossa Senhora das Graças e Departamento de Neurocirurgia do Hospital de Clínicas da Uinversidade Federal do Paraná (UFPR), Curitiba PR, Brasil: ${ }^{1}$ Residente em Neurocirurgia do Hospital Nossa Senhora das Graças; ${ }^{2}$ Neurocirurgião; ${ }^{3}$ Neurocirurgião, Preceptor da Residência em Neurocirurgia do Hospital de Clínicas; ${ }^{4}$ Neurocirurgião, Professor Adjunto do Departamento de Neurocirurgia da UFPR e Preceptor da Residência em Neurocirurgia do Hospital Nossa Senhora das Graças; ${ }^{5}$ Professor Titular do Departamento de Neurocirurgia da UFPR.
}

Recebido 11 Janeiro 2002, recebido na forma final 19 Abril 2002. Aceito 6 Maio 2002. 


\section{MÉTODO}

Característica dos pacientes - Entre 1993 e 1999, 35 pacientes foram submetidos a microcirurgia para ressecção de processo expansivo intramedular no Serviço de Neurocirurgia do Hospital de Clínicas de Curitiba - PR e no Serviço de neurocirurgia do Hospital Nossa Senhora das Graças de Curitiba - PR. Foram excluídos do estudo pacientes que não apresentavam investigação pré-operatória adequada com RM, pacientes que não tenham sido manejados cirurgicamente pelos serviços supracitados, e pacientes que não tenham sido submetidos à RM no seguimento. Os dados foram obtidos através da análise retrospectiva dos prontuários e dos exames de imagem dos pacientes. A população de pacientes foi constituída de 11 mulheres $(31,4 \%)$ e 24 homens $(68,6 \%)$. A idade no momento do diagnóstico variou de 8 a 76 anos (média 32,9 anos). A localização da lesão foi cervical em 9 casos (25,7\%), cervicotorácica em 9 (25,7\%), torácica em 14 (40\%), toracolombar em 1 (2,9\%) e lombar em 2 (5,7\%). A extensão tumoral variou de 1 a 16 segmentos vertebrais, com média de 4,3 níveis.

Avaliação pré-operatória - Todos os pacientes foram submetidos a exame neurológico pré-operatório, com avaliação da função sensitiva, motora e esfincteriana. Todos os pacientes foram submetidos a RM para avaliação diagnóstica e planejamento cirúrgico. Eventualmente a mielografia e a mielotomografia pré-operatória também foram utilizadas.

Técnica cirúrgica - Com o paciente na posição prona, é realizada incisão na linha média centrada no nível do tumor, expondo-se uma vértebra acima e uma abaixo da lesão. Quando necessária a realização de extensa laminec- tomia, é recomendada a preservação de um arco posterior a cada 4 vértebras. Em crianças, está indicada a realização de laminotomia para a prevenção de deformidades espinhais. A abertura da dura-máter é realizada com a visualização apropriada dos limites superiores e inferiores da lesão. Na ressecção tumoral, o uso do microscópio cirúrgico e de ultra-som auxiliam na identificação de alterações de coloração, componentes císticos e parâmetros anatômicos. O aspirador ultra-sônico é empregado frequentemente nestas lesões. Tenta-se identificar um plano de clivagem entre o tumor e a medula espinhal. Quando o tumor apresenta característica infiltrante, deve-se ter cuidado para não criar um falso plano de clivagem, pois a distinção entre tumor e medula espinhal normal através da coloração e da consistência pode algumas vezes ser extremamente difícil. O objetivo é a ressecção total do tumor, evitando-se lesão de tecido medular normal e consequente dano neurológico ao paciente. Nos casos em que a ressecção total foi possível, pontos de aproximação na pia-máter reconstituem a solução de continuidade provocada pela incisão. Na maioria dos casos a dura-máter foi fechada sem enxerto.

Avaliação pós-operatória - Todos os pacientes incluídos no estudo foram submetidos a RM no período de acompanhamento pós-operatório, para avaliação do grau de ressecção tumoral. $O$ estado funcional dos pacientes foi avaliado através de exame neurológico entre 6 meses e um ano do pós-operatório, classificando-se o estado neurológico do paciente como melhor, pior ou estável, de acordo com a função sensitiva, motora e esfincteriana verificada no pré-operatório.

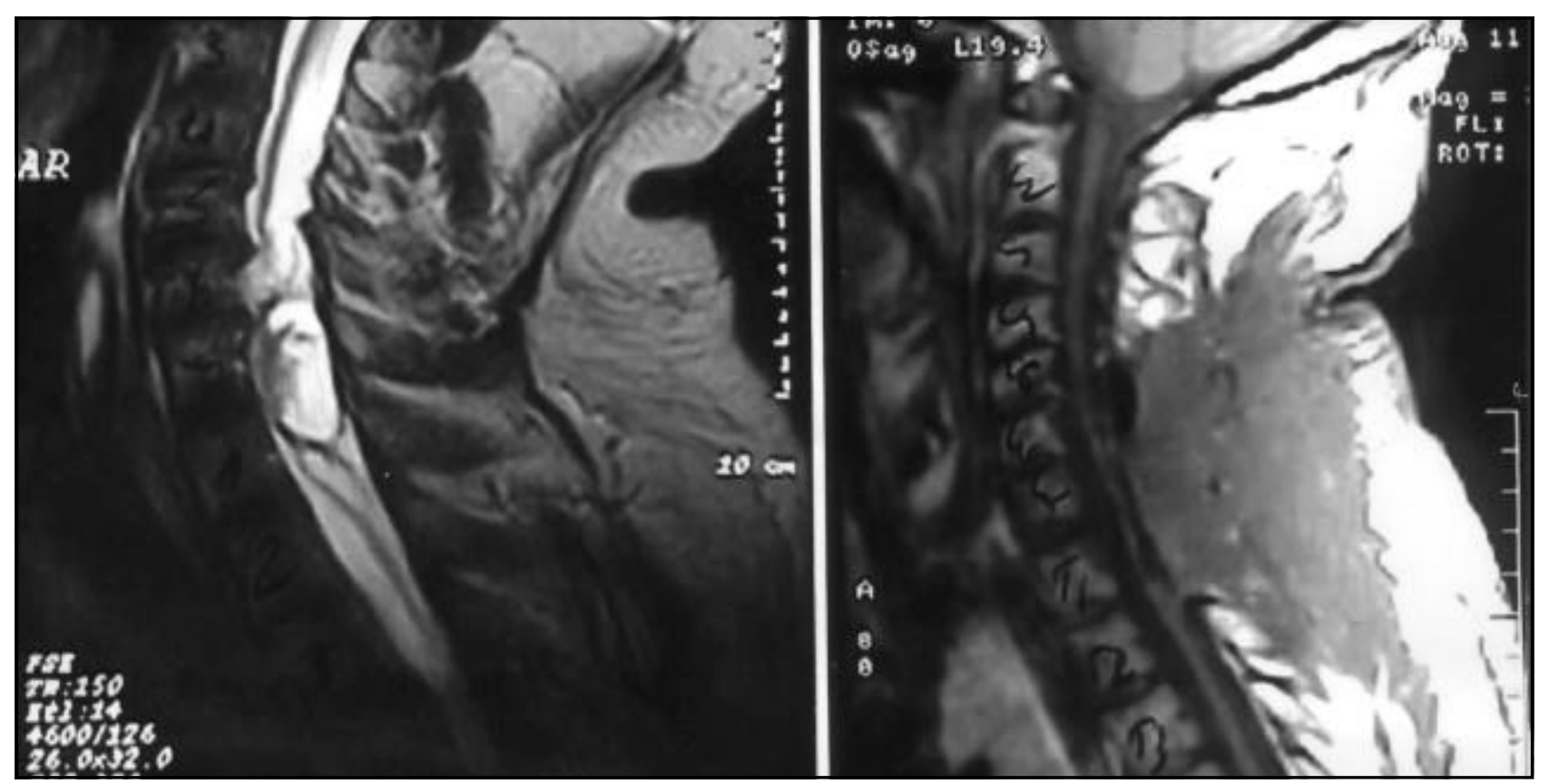

Fig 1. Ressonância magnética pré e pós-operatória de um paciente com ependimoma com ressecção completa: A. Ressonância magnética da coluna cervical pré-operatória em T2, mostrando lesão expansiva heterogênea intramedular, hiperintensa, com centro geométrico em C7-T1. B. Ressonância pós-operatória em T1, mostrando laminectomia de C4 a T1, com ressecção completa do tumor. 
A analise estatística foi realizado pelo teste para avaliação paramétrica, teste $\chi^{2}$ e Fisher quando necessário para avaliação não-paramétrica. A diferença foi considerada significativa quando o $p$ atingiu o valor de 0,05.

Radioterapia - Apenas um paciente com diagnóstico de glioblastoma foi submetido a radioterapia. Os demais tumores (gliomas de baixo grau e demais tipos histológicos) foram acompanhados sem terapia adjuvante.

\section{RESULTADOS}

Histologia - Os tipos histológicos mais frequentes foram ependimoma em 17 casos $(48,6 \%)$ e astrocitoma em 12 (34,3\%). Os demais tipos histológicos foram: cavernoma, ganglioglioma, glioblastoma, fibrolipoma e hemangioblastoma com 1 caso cada (2,9\%). Um caso de Paracoccidioides brasiliensis que inicialmente tivera diagnóstico de tumor intramedular por exame de imagem foi excluído.

Morbidade e mortalidade - O paciente com diagnóstico de glioblastoma faleceu 10 meses após cirurgia por progressão tumoral. Não houve quadro de mortalidade relacionada diretamente ao procedimento cirúrgico.

A evolução neurológica dos pacientes observada no período entre 6 meses e um ano do pós-operatório foi de melhora em 15 pacientes (42,9\%), estabilidade em relação ao pré-operatório em 12 (34,3\%) e de piora em 8 (22,9\%). Para análise dos dados referentes a fatores de morbidade no pós-operatório, os pacientes melhores e estáveis foram reunidos no grupo de "Evolução Favorável", enquanto que os pacientes de apresentaram piora pós-operatória foram reunidos no grupo de "Evolução Desfavorável".

Grau de ressecção - Foram considerados: um grupo de ressecção total, nos pacientes que a RM não demonstrou resíduo tumoral no pós-operatório (Figs 1 e 2 ), um grupo de resseção subtotal, nos pacientes em que se demonstrou resíduo tumoral à RM ou se verificou na cirurgia fragmentos macroscópicos do tumor remanescentes na parede medular, com ressecção tecnicamente inviável. O terceiro grupo foi submetido apenas a biópsia para diagnóstico da lesão.

A ressecção foi total em 20 pacientes $(57,2 \%)$ e subtotal em 13 (37,1\%). Em 2 pacientes (5,7\%), foi realizada apenas biópsia para diagnóstico histológico.

Fatores relacionados a ressecção e morbidade cirúrgica - Os pacientes portadores de ependimoma tiveram grau de ressecção total maior em relação ao grupo dos pacientes portadores de astrocitoma, com diferença estatística $(p=0,05)$, conforme Tabela 1 .

Entretanto, o grupo com ressecção total não apresentou maior déficit pós-operatório em relação aos pacientes com ressecção subtotal $(p=0,52)$, e não houve diferença entre o grupo dos ependimomas e dos astrocitomas em relação à evolução entre os grupos de evolução favorável ou desfavorável $(p=0,17)$.

A localização torácica apresentou pior evolução neurológica em relação a outros níveis medulares (Tabela 2); de 8 pacientes que apresentaram piora pós-operatória, 6 encontravam-se na região torácica $(p=0.021)$.

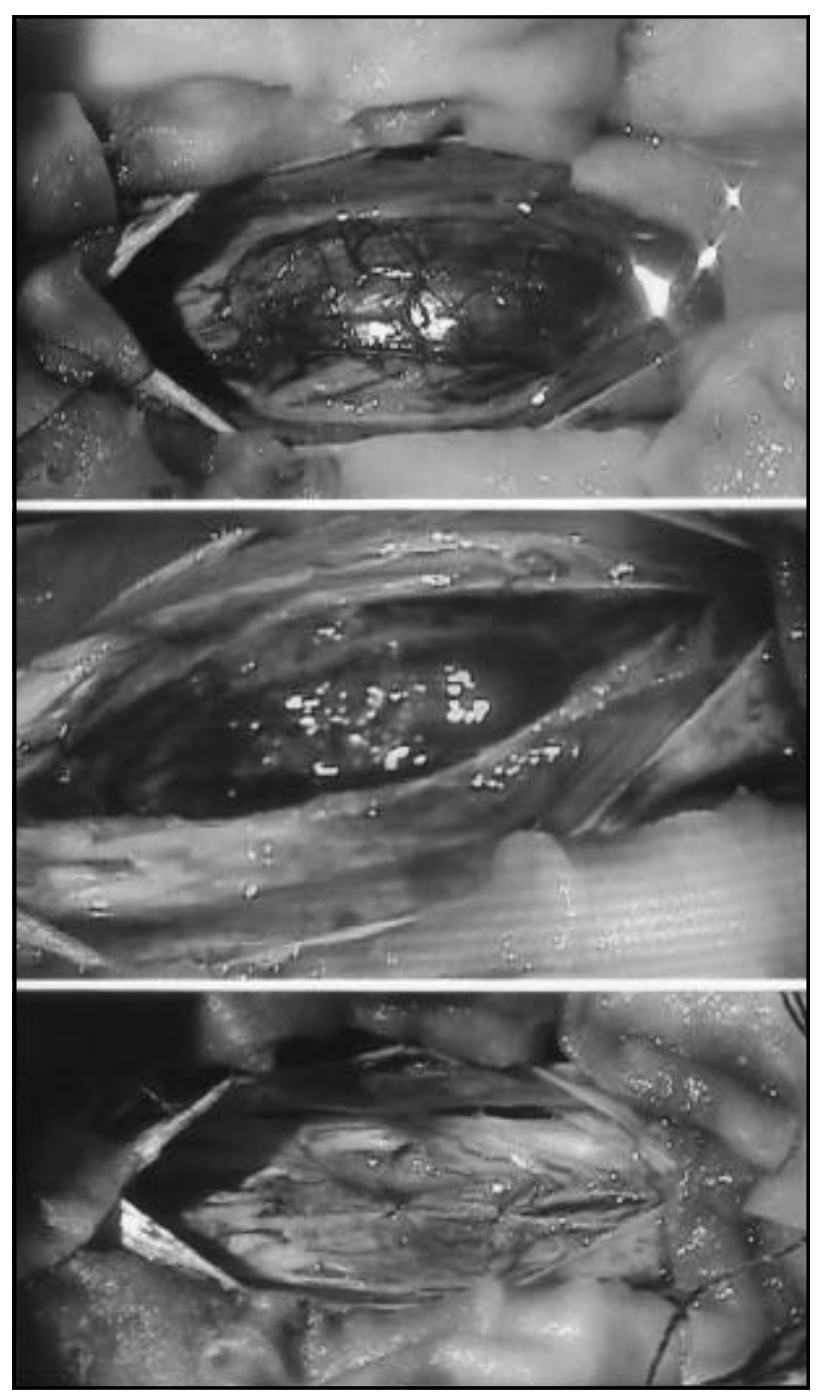

Fig 2. Fotos transoperatórias do paciente com a ressonância magnética demonstrada na Figura 1. A. Após mielotomia posterior, verifica-se a exposição de um ependimoma, com plano de clivagem entre o tumor e o tecido medular circunjacente. B. Cavidade deixada pelo tumor após ressecção completa da lesão. C. Sutura da pia-máter para reconstituição da solução de continuidade deixada pela incisão. 
Tabela 1. Relação tipo histológico x grau de ressecção.

\begin{tabular}{lcccc}
\hline $\begin{array}{l}\text { Tipo } \\
\text { Histológico }\end{array}$ & $\begin{array}{c}\text { Ressecção } \\
\text { total }\end{array}$ & $\begin{array}{c}\text { Ressecção } \\
\text { subtotal }\end{array}$ & Biópsia & Total \\
\hline Ependimoma & 13 & 3 & 1 & 17 \\
Astrocitoma & 5 & 6 & 1 & 12 \\
Total & 18 & 9 & 2 & 29 \\
\hline
\end{tabular}

Tabela 2. Relação localização x evolução pós-operatória.

\begin{tabular}{lccc}
\hline Localização & Desfavorável & Favorável & Total \\
\hline Cervical & 2 & 7 & 9 \\
Cervico-Torácico & 0 & 9 & 9 \\
Torácico & 6 & 8 & 14 \\
Tóraco-lombar & 0 & 1 & 1 \\
Lombar & 0 & 2 & 2 \\
Total & 8 & 27 & 35 \\
\hline
\end{tabular}

Na relação idade e grau de ressecção, houve maior grau de ressecção total nos jovens (média de idade de 30,6 anos), com relação à maior realização de biópsia nos idosos (média de idade de 51 anos), com significância estatística $(p=0,038)$.

Outros fatores como idade, sexo e tamanho do tumor não demonstraram correlação estatística com a morbidade pós-operatória.

\section{DISCUSSÃO}

A maioria dos pacientes com tumores intramedulares demonstrou algum grau de piora do déficit neurológico durante o período pós-operatório imediato. Isto pode ser atribuído a edema causado por manipulação cirúrgica e interferência transitória no fluxo sanguíneo da medula espinhal ${ }^{9}$. Estes novos sintomas tendem a regredir com o decorrer do tempo, com melhora da função sensitiva referida até três meses de pós-operatório, e melhora da função motora até um ano após a cirurgia ${ }^{4}$. A comparação, entre o quadro pré e pós-operatório, com melhora de cerca de $43 \%$ dos pacientes, não difere substancialmente das séries apresentadas na literatura ${ }^{3,4,11,12}$. A piora no segmento pós-operatório pode ser resultado de sequela da manipulação cirúrgica, crescimento ou eventual recidiva tumoral ${ }^{3-6}$.

O grau de ressecção foi total em cerca de dois terços dos casos, sendo significativamente maior nos ependimomas ( 13 de 17) e menor nos astrocitomas (5 de 12), fato este já bem estabelecido, devido muitas vezes a dificuldade de encontrar plano de clivagem nos astrocitomas pela sua natureza infiltrativa ${ }^{3,4,10,11,13,14}$. Entretanto, mesmo com menor grau de ressecção nos astrocitomas, estes não diferem significativamente na morbidade posterior à cirurgia em relação aos ependimomas, fatos concordantes com alguns trabalhos da literatura ${ }^{8,11,13}$ e discordante de outros, que relatam menor morbidade nos ependimomas 5 . Embora a ressecção total do tumor possa possivelmente curar o paciente, uma descompressão mecânica adequada da medula espinhal pode ser de relevância funcional para a melhora neurológica do paciente ${ }^{11}$. Segundo Samii e Klekamp ${ }^{8}$, o grau de ressecção tumoral dos astrocitomas tem pouca influência na evolução pós-operatória. Mesmo com uma ressecção parcial na maioria dos astrocitomas da sua série, apenas $18 \%$ dos casos apresentaram nova expansão, indicando que, embora a ressecção total deva ser tentada, não se deve forçar ressecção radical sem plano de clivagem identificável, sob o risco de importantes danos pósoperatórios ao paciente. Em nosso grupo de astrocitomas, de 6 pacientes com ressecção subtotal, apenas 1 apresentou evolução desfavorável.

Pacientes com déficit neurológico avançado no pré-operatório apresentam risco maior de deterioração no pós-operatório, fato este que torna a intervenção cirúrgica precoce recomendada ${ }^{8,9,11-13,15,16}$.

Gliomas medulares de alto grau apresentam mau prognóstico em relação à evolução neurológica e à sobrevida ${ }^{3,5,11,14}$. Parece não haver nenhum fator de tratamento que altere significativamente a evolução destes tumores ${ }^{17,18}$. Entretanto, não foi possível comprovar este dado estatisticamente neste trabalho por haver apenas um caso de glioma maligno na nossa série (um glioblastoma).

Uma pior evolução foi verificada em tumores localizados na região torácica, fato este relatado por poucos autores ${ }^{9,11}$. O fato da medula torácica apresentar vascularização limítrofe em alguns níveis pode justificar maior vulnerabilidade desta região à manipulação de tumores neste local ${ }^{9,19,20}$.

A predominância de ressecções totais em pacientes jovens e de biópsias em pacientes mais idosos pode justificar-se pela tendência de ser o cirurgião menos agressivo em pacientes de maior idade, bem como pela dificuldade de valorização deste dado do ponto de vista estatístico, já que apenas 2 pacientes foram submetidos a biópsia.

As demais variáveis como idade, sexo e tamanho da lesão não demonstraram importância na morbidade cirúrgica, igualmente descrito em outras séries ${ }^{9,15}$. 


\section{CONCLUSÃO}

Tumores intramedulares devem ser operados o mais precocemente possível, visto que a condição neurológica pré-operatória é fundamental na evolução pós-operatória do paciente.

Os ependimomas possibilitam grau de ressecção cirúrgica maior que os astrocitomas. Este fato, no entanto, não alterou a morbidade cirúrgica quando comparados estes dois grupos tumorais.

Pacientes com ressecção subtotal de tumores não malignos podem permanecer estáveis por um longo período de tempo ${ }^{8}$. Nestes casos não há indicação de radioterapia complementar ${ }^{4,21}$ e a reintervenção como método alternativo deve ser tentada ${ }^{4}$. 0 comportamento biológico deste subgrupo necessita de maior tempo de seguimento para ser melhor avaliado.

A localização tumoral na região torácica apresenta tendência de pior evolução quando comparada a outras localizações medulares, provavelmente devido a peculiaridades anatômicas e vasculares da região.

Outros fatores como idade, sexo e tamanho tumoral não influenciaram na evolução pós-operatória.

\section{REFERÊNCIAS}

1. ClemenceauS, Lopez M. Intramedullary tumors. Rev Prat 2001;51:1206-1210.

2. Naidu MR, Dinakar I. Intramedullary mass lesions of the spinal cord. Clin Neurol Neurosurg 1989; 91:135-138.

3. Fornari M, Pluchino F, Solero CL, et al. Microsurgical treatment of intramedullary spinal cord tumors. Acta Neurochir (Wien) 1988;43(Suppl):3-8.

4. Fischer G, Brotchi J. Results . In Fischer G, Brotchi J (eds). Intramedullary spinal cord tumors. New York: Thieme, 1996:85-104
5. Houten JK, Weiner HL. Pediatric intramedullary spinal cord tumors: special considerations. J Neurooncol 2000;47:225-230.

6. Miller DC. Surgical pathology of intramedullary spinal cord neoplasms. J Neurooncol 2000;47:198-194.

7. McCormick PC, Stein BM. Microsurgical approaches to cervical spine. In Young PH (ed). Microsurgery of the cervical spine. New York: Raven Press, 1991:159-174.

8. Samii M, Klekamp J. Surgical results of 100 intramedullary tumors in relation to accompanying syringomyelia. Neurosurgery 1994;35:865-873.

9. Hoshimaru M, Koyama T, Hashimoto N, Kikuchi H. Results of microsurgical treatment for intramedullary spinal cord ependimomas: analysis of 36 cases. Neurosurgery 1999;44:264-269.

10. Sandler HM, Papadopoulos SM, Thornton AF Jr, Ross DA: Spinal cord astrocytomas: results of therapy . Neurosurgery 1992;30:490-493.

11. Cristante L, Herrmann HD. Surgical management of intramedullary spinal cord tumors: funcional outcome and sources of morbity. Neurosurgery 1994;35:69-76.

12. Cooper PR, Epstein F. Radical resection of intramedullary spinal cord tumors in adults: recent experience in 29 patients. J Neurosurg 1985;63:492-499.

13. Innocenzi G, Raco A, Contore G, Raimondi AJ. Intramedullary astrocytomas and ependymomas in the pediatric age group: a retrospective study. Childs Nerv Syst 1996;12:776-780.

14. Fischer G. Spinal cord astrocytomas and ependymomas: therapeutic strategy. Chirurgie 1997;122:127-129.

15. Ferrante L, Mastronardi L, Celli P, Lunardi P, Acqui M, Fortuna A. Intramedullary spinal cord ependymomas: a study of 45 cases with long- term follow-up. Acta Neurochir (Wien) 1992;119:74-79.

16. Epstein FJ, Farmer JP, Freed D. Adult intramedullary spinal cord ependymomas: the result of surgery in 38 patients. J Neurosurg 1993;79:204-209.

17. Kopelson G, Linggood RM. Intramedullary spinal cord astrocytomas versus glioblastoma: the prognostic importance of histologic grade. Cancer 1982;50:732-735.

18. Linstadt DE, Wara WM, Leibel S A, Gutin PH, Wilson CB, Sheline GE. Postoperative radiotherapy of primary spinal cord tumors. Int J Radiat Oncol Biol Phys 1989;16:1397-1403.

19. Maiman DJ, Pintar FA. Anatomy and clinical biomechanics of the thoracic spine. Clin Neurosurg 1992;38:296-324.

20. Stillerman CB, Weiss MH. Management of thoracic disc disease. Clin Neurosurg 1992;38:325-352.

21. Truite LVR, Hanel RA, Grande CV, Torres LFB, Araújo JC. Ganglioglioma da medula espinhal: relato de caso. Arq Neuropsiquiatr 2001;59:431-434. 